\title{
Corrigendum
}

\section{Developmental pathways to social anxiety and irritability: The role of the ERN - CORRIGENDUM}

\author{
Courtney A. Filippi, Anni R. Subar, Jessica F. Sachs, Katharina Kircanski, George Buzzell, David Pagliaccio, Rany Abend, \\ Nathan A. Fox, Ellen Leibenluft and Daniel S. Pine
}

doi: https://doi.org/10.1017/S0954579419001329. Published online by Cambridge University Press, 28 October 2019

Keywords: behavioral inhibition, developmental pathways, ERN, irritability, psychopathology, corrigendum

The article "Developmental pathways to social anxiety and irritability: The role of ERN" (Filippi et al., 2020) included an error.

The authors identified an error in one of the scripts used to process the event-related potential (ERP) data in the original manuscript (Buzzell et al., 2017). A typo in one of the MATLAB scripts caused one of the seven electrodes going into the error-related negativity (ERN) ERP cluster to be from an incorrect scalp location (computing the cluster relies on indexing into a matrix and one of the indices were off by a value of 1 ). This mistake ultimately impacts the ERN variable described in the original article (Filippi et al., 2020). Given that only one of the 7 electrodes in the cluster was wrong, after correcting this error, the original and corrected ERN variables-for the sample used in this paper-correlate highly ( $\mathrm{n}=127, r=.99, p<.001)$. Similarly, after re-running all analyses in the main text and supplement using the corrected variable, we find that all primary results involving the ERN (interactions, follow-up correlations, and partial correlation tests) remain unchanged in terms of significance. It is worth noting that while one of the control analyses does change from $p<.05$ to $p=.06$, the associated follow-up partial correlation tests remain unchanged in terms of significance. Thus, there are no changes in the interpretation or conclusions drawn from the manuscript as a result of correcting the error with the ERN variable. Please note that this is an author-initiated correction, in line with the principles of open science and to ensure the validity of any future metaanalytic work based on this manuscript. Changes to the originally published article and supplement are listed below in red font.

1. Page 901, Table 2 should appear as follows:

2. Page 901, "Focal analysis strategy" section, paragraph 1, lines 11-14 should read:

"High ERN reflects scores less than $-3.28(n=42)$; moderate ERN reflect scores that are between -3.28 and $-.98(n=43)$; and low ERN reflects scores that are greater than $-.98(n=42)$ "

Cite this article: Filippi CA et al (2022). Developmental pathways to social anxiety and irritability: The role of the ERN - CORRIGENDUM. Development and Psychopathology 34: 1198-1200, https://doi.org/10.1017/S0954579421000316
3. Page 901, "Behavioral inhibition to social anxiety" section, paragraph 1, lines 1-4 should read:

"Results indicate distinct correlations between ERN and social anxiety in children with and without BI, $\beta=-.083, \Delta R^{2}=.027$, $F(1,122)=4.037, p<.047^{\prime \prime}$

4. Page 902, Figure 2 should appear as follows:

5. Page 902, "Behavioral inhibition to social anxiety" section, paragraph 2, lines 6-14 should read:

"Results indicated that the association between BI and social anxiety was significant for the high-ERN, $r(41)=.563, p<.001$, and the moderate-ERN groups, $t(41)=.521, p<.001$, but not the low-ERN group $(p>$.786). This suggests that the association between BI and social anxiety found in the two higher ERN groups fails to manifest among individuals who exhibit a low ERN. These correlations held when controlling for childhood irritability, high-ERN: $r(36)=.547, p<.001$; moderate-ERN: $r(39)=.533$, $p<.001$; low-ERN: $p<.565)$."

6. Page 902, "Childhood irritability to irritability at age 12" section, paragraph 1 , lines 1-4 should read:

The association between childhood irritability and age-12 irritability differed as a function of ERN magnitude, $\beta=.590, \Delta R^{2}=.078$, $F(1,59)=5.806, p<.019 "$

Table 2. Correlations among focal variables of interest

\begin{tabular}{|c|c|c|c|c|c|}
\hline & (1) & (2) & (3) & (4) & (5) \\
\hline (1) Childhood BI & - & - & - & - & - \\
\hline $\begin{array}{l}\text { (2) High stable irritability } \\
\text { childhood }\end{array}$ & .070 & - & - & - & - \\
\hline (3) ERN & .024 & .049 & - & - & - \\
\hline (4) 12-year Social Anxiety & $.341^{\star \star}$ & -.031 & .024 & - & - \\
\hline (5) 12-year Irritability (ARI) & -.035 & $.350^{\star \star}$ & -.142 & .190 & - \\
\hline
\end{tabular}


Table 3.

\begin{tabular}{|c|c|c|c|c|c|c|}
\hline Model & Predictor & $\beta$ & SE B & $t$ & $p$ & Fit \\
\hline \multirow{2}{*}{1} & ERN & -.009 & .023 & -.371 & .711 & \\
\hline & $\mathrm{BI} \times \mathrm{ERN}$ & -.083 & .041 & -2.009 & .047 & \\
\hline \multirow{2}{*}{2} & $\mathrm{BI} \times \mathrm{ERN}$ & -.080 & .042 & -1.902 & .060 & \\
\hline & High childhood irritability & -.024 & 214 & -.112 & 911 & \\
\hline
\end{tabular}

Regression results predicting social anxiety at age 12

Note: As reported in the original article, the primary interaction between $\mathrm{BI}$ and ERN does not change in significance (Model 1 ) and all follow-up correlation tests probing the nature of this interaction for Model 1 do not change in significance. In Model 2, when controlling for irritability, the BI x ERN changes from $p<.05$ to $p=.06$. Nonetheless, all follow-up partial correlation tests probing the nature of this interaction in Model 2 do not change in significance.

Table 4.

\begin{tabular}{|c|c|c|c|c|c|c|}
\hline Model & Predictor & $\beta$ & SE B & $t$ & $p$ & Fit \\
\hline \multirow{2}{*}{1} & ERN & -.139 & .065 & -2.127 & .038 & \\
\hline & High childhood irritability $x$ ERN & .590 & .245 & 2.41 & .019 & \\
\hline \multirow{2}{*}{2} & High childhood irritability $x$ ERN & .597 & .247 & 2.421 & .019 & \\
\hline & $\mathrm{BI}$ & .180 & .327 & .551 & .584 & \\
\hline
\end{tabular}

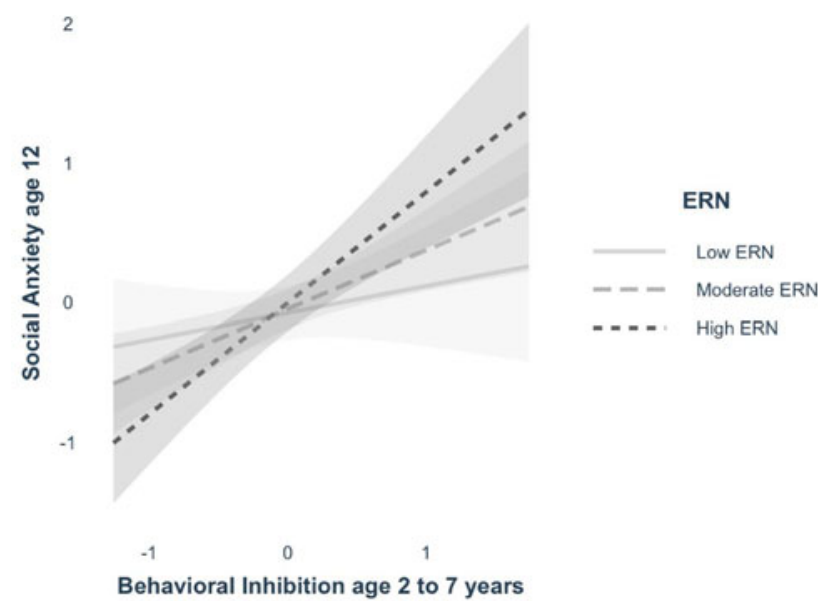

Figure 2.

7. Page 902, "Childhood irritability to irritability at age 12 " section, paragraph 2, lines 5-10 should read:

"Results indicated that the correlation between childhood and age-12 irritability did manifest in the low-ERN group, $r(23)$ $=.613, p<.002$, but not in the high- or moderate-ERN groups (all ps> .05). These results held when controlling for BI, low-ERN: $r(20)=.615, p<.002$; moderate- and high-ERN, $p s>$ .09."

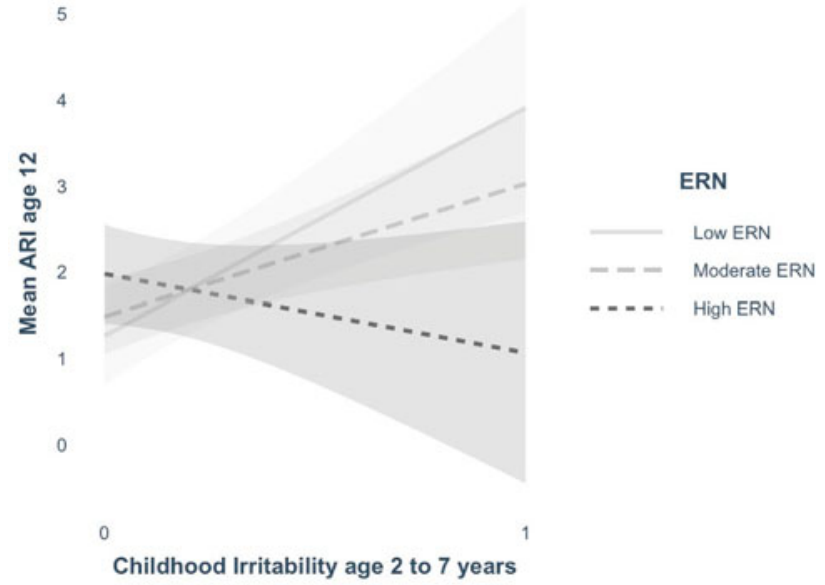

Figure 3.

8. Page 903, Table 3 should appear as follows:

9. Page 903, Figure 3 should appear as follows:

10. Page 904, Table 4 should appear as follows:

Supplementary Material. The supplementary material for this article can be found at https://doi.org/10.1017/S0954579421000316 


\section{References}

Buzzell, G. A., Troller-Renfree, S. V., Barker, T. V., Bowman, L. C., Chronis-Tuscano, A., Henderson, H. A., Kagan, J., Pine, D. S., \& Fox, N. A. (2017). A Neurobehavioral Mechanism Linking Behaviorally Inhibited Temperament and Later Adolescent Social Anxiety. Journal of the American Academy of Child and Adolescent Psychiatry, 56(12), 10971105. doi:10.1016/j.jaac.2017.10.007

Filippi, C. A., Subar, A. R., Sachs, J. F., Kircanski, K., Buzzell, G., Pagliaccio, D., Abend, R., Fox, N. A., Leibenluft, E., \& Pine, D. S. (2020). Developmental pathways to social anxiety and irritability: The role of the ERN. Development and Psychopathology, 32(3), 897-907. doi:10.1017/S0954579419001329 